Original article

\title{
Pulmonary physiotherapy effect on patients undergoing open cardiac surgery
}

\author{
Seyed Kazem Shakuri, Yaghoub Salekzamani, Ali Taghizadieh, Hamed Sabbagh-Jadid, Jamal Soleymani, \\ Leyla Sahebi
}

Tabriz University of Medical Science, Tabriz, Iran

Received 23 May 2014, Accepted 11 August 2014

(C) 2014, Shakuri S.K., Salekzamani Y., Taghizadieh A., Sabbagh-Jadid H., Soleymani J., Sahebi L.

(C) 2014, Russian Open Medical Journal

\begin{abstract}
Backgrounds and Objectives - Respiratory complications after open heart surgeries are common problems which can lead to death if not properly managed. The aim of this study was to evaluate the role of pulmonary rehabilitation before and after surgery for reducing the risk of pulmonary complications after surgery also correlations of the six-minute walk test and respiratory following open heart surgery. Material and Methods - In a randomized clinical trial, 60 patients undergoing heart surgery were divided into two groups randomly (groups A and B). In group A it was performed physiotherapy before and after chest physiotherapy surgery, but on patients in group B were done only chest physiotherapy after surgery. Effects of preoperative pulmonary rehabilitation were compared between two groups, using spirometry and six-minute walk test. Results - Thirty nine of males (65\%) and 21 females (35\%) with a mean age of $8.10 \pm 9.56$ was been analyzed. The mean difference in predicted forced vital capacity (Cl95\%: 1.3 to 8.7) and predicted peak flow indices (CI95\%: 1.9 to 9.4) of spirometery indicator was significant, also evaluation of six-minute walk test showed, mean difference in walking distant (CI95\%: 8.8 to 21.0) and mean oxyhemoglobin saturation (CI95\%: 0.59 to 1.67) in group A was more than group B. Inverse correlation of heart rate with forced vital capacity showed that patients with more restriction had more heart rate during the walking test. Conclusion - Pulmonary rehabilitation program before surgery is recommended to reduce complications of heart surgery. Further evaluations are necessary in relation to the sensitivity and specificity of six-minute walk test parameters alone in the evaluation of respiratory performance.
\end{abstract}

Keywords: cardiac surgery, movement techniques, breathing exercises, randomized clinical trial, six-minute walk test, spirometry

Cite as Shakuri SK, Salekzamani Y, Taghizadieh A, Sabbagh-Jadid H, Soleymani J, Sahebi L. Pulmonary physiotherapy effect on patients undergoing open cardiac surgery. Russian Open Medical Journal 2014; 3: 0306.

Correspondence to Hamed Sabbagh-Jadid. E-mail: dr.sabbagh@ymail.com. Telefax: 098-0411-3378093.

\section{Introduction}

Cardiac surgery is associated with pulmonary complications (PC) incidence, defined any pulmonary abnormality that occurs during the postoperative period which creates detectable syndrome that is clinically important and affects the clinical course [1]. The global average prevalence of PC following coronary arterial bypass graft (CABG) surgery was estimated between $2-4 \%$ [2]. The most common complications was reported, atelectasis (27-95\%), pleural effusion (16.6-88.0\%) and phrenic nerve paralysis $(30-75 \%)$ [3]. Possibility of PC resulting the CABG in Iran not accessible but the event of PC following the public surgery has been reported 50\% [4-5]. PC following cardiac surgery leads to chief socio-economic burden such as prolonging hospitalization, intensive care units (ICU) admission, the huge cost of treatment, loss of the work days and even death [1]. Some thoracic physiotherapy techniques are used in order to Respiratory Functionim provement in oxygenation and decline in respiratory complications incidence after CABG [7]. Despite the common use of respiratory exercises in patients after CABG in different countries, there is still insufficient scientific evidence for their efficacy [8] furthers more how to assess respiratory function is unclear. Spirometry is invaluable as a screening test of general respiratory health in the same way that blood pressure provides important information about general cardiovascular health [8] and as well as national guidelines, advise spirometry as the gold standard for accurate and repeatable measurement of lung function. The six-minute walk test (6MWT) can be easily performed, and associated parameters during 6MWT could provide important indicators for treatment interpretation [9].

The objective of this study was survey of pre\& post-operative respiratory rehabilitation in patients undergoing open cardiac surgery based spirometry and 6MWT in addition correlation between spirometry and 6MWT [9].

\section{Material and Methods}

\section{Design}

The patients were asked to take part in the study after they received full explanations about the aim of the study, random allocation to study groups and reassurance about the absence of any side effects. The study protocol was approved by the local Ethics Committee (with a code of 2272/4/5) and was registered in the International Center for Clinical Trials with a code of IRCT138904294422N1. The patients were randomly assigned to the intervention (Group 1) and control (Group 2) groups. 
Collection of samples took approximately 12 months. The reliability of the study was evaluated and confirmed by a physiotherapist and an evaluator who had been trained by the researcher. The equipment used for the physiotherapy and evaluation of the respiratory function was standard and was used under the direct supervision of the principal investigator in the present study.

The patients in group A underwent physiotherapy 15 days before the surgical operation, with an emphasis on strengthening inspiratory muscles, and thoracic cavity physiotherapy was carried out based on the surgical ward routines. However, patients in group B received only post-operative physiotherapy based on surgical ward routines.

\section{Participants}

Sixty seven patients were candidates for open cardiac surgery using the mid-sternotomy technique in Tabriz Cardiac Educational Treatment Center and had similar conditions in relation to the administration of medicines. A total of 60 CABG patients were included in the present randomized clinical trial in a period of 12 months (2011-2012). The exclusion criteria consisted of a history of any chronic respiratory condition, emergency surgery, cardiac insufficiency $(\mathrm{EF}<40 \%)$, valvular disorders, a history of connective tissue conditions, chronic renal insufficiency and a history of musculoskeletal conditions.

\section{Intervention}

Pre-operative physiotherapy techniques for group A consisted of the following:

1. Breathing exercises consisting of 10 deep breathing attempts, diaphragmatic breathing and pursing of the lips;

2. Instruction of flow-incentive spirometer-based (Respiflow ${ }^{\mathrm{TM}}$ FS) and effective coughing;

3. Instruction of neck and shoulder mobilization exercises with an emphasis on thoracic extension and rotation;

4. Instruction of muscular tension exercises;

5. Instruction of exercises to strengthen muscles responsible to move the shoulders forwards and backwards.

Post-operative exercises and physiotherapy procedures in groups $A$ and $B$ were carried out as follows:

1. Techniques to cleanse the lungs including mobilization, manual techniques, use of the active cycle ofbreathing techniques and use of incentive spirometer;

2. Auxiliary active movements of the extremities;

3. Active movement of the extremities;

4. Breathing exercises and expansion of the lobes of the lungs.

In group A during the two-week period before the surgical operation, 15 sessions were held, consisting of exercises and auxiliary activities for extension and rotation of thoracic vertebrae, breathing exercises, exercises to expand lung lobes, instruction of incentive spirometer equipment, extension exercise for thoracic cavity muscles and muscles with a role in breathing (aerobic exercises) for 25 minutes at a constant low speed forall the patients. The patients once again underwent physiotherapy for the respiratory system. However, patients in group B, received physiotherapy only after surgery.

\section{Outcome measures}

Evaluation of respiratory function was carried out by trained physiotherapists, using 2 spirometry parameters and 6MWT, in group A, spirometry and 6MWT were carried out 15 days before surgery and immediately after discharge from the ICU. In group B, too, spirometry and 6MWT were carried out before surgery and immediately after discharge from the ICU, and the parameters were compared.

Spirometry parameters included forced expiratory volume in 1 second (predicted FEV1), predicted forced vital capacity (FVC) and peak expiratory flow (PEF). Spirometry was carried out in group $A$ (intervention) once before rehabilitation programs and once after discharge from the ICU. Butin group B once 15 days before CABG and once immediately after discharge from the ICU.

6MWT parameters consisted of heart rate, the distance walked, maximum oxyhemoglobin saturation ( $\left.\mathrm{Max} \mathrm{spO}_{2}\right)$, minimum oxyhemoglobin saturation $\left(\mathrm{Min} \mathrm{spO}_{2}\right)$ and mean oxyhemoglobin saturation (Mean $\mathrm{spO}_{2}$ ).

6MWT was carried out in all the patients in three 2-minute stages as follows:

1. The initial stage: two minutes of rest on a chair;

2. The second stage: walking for 2 minutes on a smooth surface;

3. The third stage: recovery at chair rest.

6MWT was carried out on each patient with the patient wearing comfortable shoes and comfortable hospital clothes without any extra loads in hands during the second stage. The patients chose the walking pace voluntarily and felt at ease at that pace.

6MWT was carried out in the intervention group (A) once before rehabilitation programs and once immediately after discharge from the ICU; the test was carried out in the control group (B) once before CABG operation and once immediately after discharge from the ICU.

In a pilot study on 10 patients, once the reliability of the observer and once the reliability of the equipment were evaluated. Kappa coefficient was used to estimate the reliability of the study, which was estimated to be more than $90 \%$ in both evaluations.

\section{Data analysis}

Student's t-test analyses and multiple linear regression were used to compare the study variables at a confidence interval of 95\%, using SPSS 18.

\section{Results}

\section{Flow of participants through the trial}

Sixty seven patients with candidates for open cardiac surgery using the mid-sternotomy technique were screened for eligibility between October 2011 to November 2012. In the present clinical trial, sixty patients were randomly assigned to groups $A(n=30)$ and $B(n=30)$. Seven patients were excluded from the study for various reasons, including arthritis, dissatisfaction and lack of access. The groups A and B had similar baseline demographic characteristics and difference between them was not statistically significant (age, $\mathrm{P}=0.096$; sex, $\mathrm{P}=0.97$; smoking, $\mathrm{P}=0.51$; diabetes mellitus, $\mathrm{P}=0.51$; body mass index $(\mathrm{BMI}), \mathrm{P}=0.29$; duration of operation, $\mathrm{P}=0.82$ ) (Table 1). 
Table 1. Baseline variables of patients who completed the study

\begin{tabular}{|c|c|c|c|}
\hline \multirow{2}{*}{ Variables } & \multicolumn{2}{|c|}{ Groups } & \multirow{2}{*}{ P-Value } \\
\hline & Expression $(n=30)$ & Control $(n=30)$ & \\
\hline Age; Years, mean \pm SD & $54.4 \pm 10.8$ & $59.3 \pm 10.45$ & 0.09 \\
\hline Gender: female, no (\%) & $11(36.7)$ & $10(33.3)$ & 0.97 \\
\hline Smoking, no (\%) & $9(30.0)$ & $10(33.3)$ & 0.51 \\
\hline Diabetes mellitus, no (\%) & $11(36.7)$ & $8(26.6)$ & 0.29 \\
\hline $\mathrm{BMI}, \mathrm{kg} / \mathrm{m}^{2}$, mean $\pm \mathrm{SD}$ & $26.8 \pm 4.7$ & $27.7 \pm 4.9$ & 0.46 \\
\hline Duration of operation, hours, mean $\pm S D$ & $2.6 \pm 0.2$ & $2.8 \pm 0.4$ & 0.82 \\
\hline
\end{tabular}

$\mathrm{SD}$, standard deviation; BMI, body mass index.

Table 2. Mean \pm SD for all outcomes for each group, Mean \pm SD difference within groups, and comparison (CI95\%) between groups

\begin{tabular}{|c|c|c|c|c|c|c|c|c|}
\hline & \multirow[t]{3}{*}{ Groups } & & & & & \multicolumn{2}{|c|}{ Difference within groups } & \multirow{3}{*}{$\begin{array}{c}\text { Difference between } \\
\text { interventions } \\
\text { Post- minus preoperative } \\
\text { Expression-Control }\end{array}$} \\
\hline & & \multicolumn{2}{|c|}{ Preoperative } & \multicolumn{2}{|c|}{ Post-operative } & \multicolumn{2}{|c|}{ Post- minus preoperative } & \\
\hline & & $\begin{array}{c}\text { Expression } \\
(n=30)\end{array}$ & $\begin{array}{l}\text { Control } \\
(n=30)\end{array}$ & $\begin{array}{c}\text { Expression } \\
(n=30)\end{array}$ & $\begin{array}{c}\text { Control } \\
(n=30)\end{array}$ & $\begin{array}{c}\text { Expression } \\
(\mathrm{n}=30)\end{array}$ & $\begin{array}{l}\text { Control } \\
(n=30)\end{array}$ & \\
\hline Spirometry & FEV1, ml & $81.7 \pm 13.1$ & $79.1 \pm 13.4$ & $80.0 \pm 12.4$ & $73.8 \pm 13.1$ & $-1.4 \pm 2.2$ & $-5.3 \pm 10.9$ & $3.9(-0.5$ to 7.9$)$ \\
\hline \multirow[t]{2}{*}{ indicators } & FVC & $85.8 \pm 9.7$ & $81.1 \pm 10.6$ & $84.5 \pm 9.0$ & $74.7 \pm 12.8$ & $-1.4 \pm 2.4$ & $-6.4 \pm 9.8$ & $5.0^{*}(1.3$ to 8.7$)$ \\
\hline & PEF & $68.3 \pm 16.0$ & $74.0 \pm 15.9$ & $68.5 \pm 14.3$ & $68.7 \pm 16.5$ & $0.2 \pm 7.2$ & $-5.5 \pm 7.2$ & $5.7 *(2.0$ to 9.4$)$ \\
\hline \multirow[t]{5}{*}{ 6MWT } & $\mathrm{HR}$, beat/min & $107.7 \pm 12.5$ & $110.0 \pm 14.5$ & $98.1 \pm 7.9$ & $101.2 \pm 9.7$ & $-9.6 \pm 7.3$ & $-8.8 \pm 9.5$ & $-0.8(-5.2$ to 3.6$)$ \\
\hline & $\begin{array}{l}\text { Walking distance, } \\
\text { meter }\end{array}$ & $110.9 \pm 20.6$ & $104.5 \pm 22.5$ & $97.7 \pm 16.39$ & $76.3 \pm 20.5$ & $-13.3 \pm 9.9$ & $-28.2 \pm 13.5$ & $14.9 *(8.8$ to 21.0$)$ \\
\hline & $\max \mathrm{spO}_{2}, \%$ & $96.1 \pm 1.5$ & $97.1 \pm 0.9$ & $96.4 \pm 5.34$ & $97.1 \pm 1.4$ & $0.3 \pm 4.9$ & $-0.03 \pm 1.0$ & $0.33(-1.5$ to 2.1$)$ \\
\hline & $\min \mathrm{spO}_{2}, \%$ & $91.4 \pm 1.9$ & $91.2 \pm 1.9$ & $92.1 \pm 2.0$ & $91.2 \pm 1.4$ & $0.7 \pm 1.2$ & $0.0 \pm 2.1$ & $0.67(-1.5$ to 0.26$)$ \\
\hline & mean $\mathrm{spO}_{2}, \%$ & $94.0 \pm 1.3$ & $94.3 \pm 1.2$ & $94.9 \pm 1.2$ & $94.1 \pm 1.2$ & $0.9 \pm 0.9$ & $-0.2 \pm 1.2$ & $1.1 *(0.6$ to 1.7$)$ \\
\hline
\end{tabular}

Table 3. Correlation distribution between spirometry and 6WMT parameters among patients after CABG

\begin{tabular}{|c|c|c|c|c|c|c|}
\hline & \multirow[t]{2}{*}{ Spirometry } & \multicolumn{5}{|c|}{$6 M W T$} \\
\hline & & $\mathrm{HR}$, beat/min & Walking distance, meter & $\mathrm{Max} \mathrm{spO}_{2}, \%$ & Min $\mathrm{spO}_{2}, \%$ & Mean $\mathrm{spO}_{2}, \%$ \\
\hline \multirow[t]{2}{*}{$\mathrm{FEV} 1, \mathrm{ml}$} & Pearson Correlation & -0.401 & 0.207 & 0.128 & -0.536 & 0.571 \\
\hline & P-level (2-tailed) & 0.002 & 0.112 & 0.331 & $<0.001$ & $<0.001$ \\
\hline \multirow[t]{2}{*}{ FVC } & Pearson Correlation & -0.558 & 0.303 & 0.130 & 0.440 & 0.513 \\
\hline & P-level (2-tailed) & $<0.001$ & 0.019 & 0.323 & $<0.001$ & $<0.001$ \\
\hline \multirow[t]{2}{*}{ PEF } & Pearson Correlation & -0.147 & 0.568 & 0.038 & 0.217 & 0.284 \\
\hline & P-level (2-tailed) & 0.263 & $<0.001$ & 0.772 & 0.096 & 0.028 \\
\hline
\end{tabular}

Table 4. Multiple regression of spirometry parameters for the six-minute walk distance

\begin{tabular}{|c|c|c|c|c|c|c|c|c|c|c|}
\hline \multirow[t]{3}{*}{ Spirometry } & \multicolumn{10}{|c|}{$6 M W T$} \\
\hline & \multicolumn{2}{|c|}{$\mathrm{HR}$, beat/min } & \multicolumn{2}{|c|}{ Walking distance, meter } & \multicolumn{2}{|c|}{$\mathrm{Max} \mathrm{spO}_{2}, \%$} & \multicolumn{2}{|c|}{ Min $\mathrm{spO}_{2}, \%$} & \multicolumn{2}{|c|}{ Mean $\mathrm{spO}_{2}, \%$} \\
\hline & $\beta$ & P-level & $\beta$ & P-level & $\beta$ & P-level & $\beta$ & P-level & $\beta$ & P-level \\
\hline $\mathrm{FEV}^{+}$ & -0.044 & 0.808 & 0.235 & 0.360 & -0.031 & 0.726 & -0.101 & 0.009 & 0.060 & 0.016 \\
\hline $\mathrm{FVC}^{++}$ & -0.693 & 0.001 & 0.345 & 0.210 & 0.038 & 0.692 & 0.017 & 0.671 & 0.026 & 0.313 \\
\hline $\mathrm{PEF}^{+++}$ & 0.079 & 0.552 & 0.856 & 0.000 & -0.009 & 0.893 & 0.003 & 0.902 & 0.010 & 0.574 \\
\hline
\end{tabular}

\section{Compliance with trial method}

All candidate CBAG patients received Pre-operative physiotherapy which they had been randomly allocated. All data were collected and analyzed as intended.

\section{Effect of intervention}

After data normality was confirmed, making it possible to use parametric tests.

In order to neutralize the effect of each patient's initial status in relation to respiratory function, Spirometry and 6MWT parameters were measured in both groups before rehabilitation and surgery. The two groups were evaluated and compared in relation to differences in each parameter before and after rehabilitation in each patient. Analysis was based t-student (Table 2). Evaluation of spirometry parameters by mean difference analysis showed significant changes in predicated FVC (Cl95\%: 1.3 to 8.7 ) and PEF (CI95\%: 1.98 to 9.4 ) in the group A compared to the group B (Table 2).
Evaluation of 6MWT showed, mean difference in walking distant (CI95\%: 8.8 to 21.0$)$ and mean $\mathrm{spO}_{2}$ (CI95\%: 0.59 to 1.67 ) in group $A$ was more than group $B$ significantly, but mean difference of $\max \mathrm{spO}_{2}$ and $\min \mathrm{spO}_{2}$ in two groups (A and $\mathrm{B}$ ) weren't significant ( $\mathrm{Cl} 95 \%$ : -1.49 to 2.1 and -1.48 to 0.26 respectively).

The Pearson correlation coefficient were calculated to examine the correlation between the 6MWT parameters and spirometry parameters in Agroup (intervention).

Table 3 shows that the heart rate $(\mathrm{HR})$ was correlated with FEV1 $(r=-0.4, P=0.002)$ and FVC $(r=-0.56, P<0.001)$ walking distance was correlated with FVC $(r=0.30, P=0.019)$ and peak flow $(r=0.57$, $\mathrm{P}<0.001)$, min $\mathrm{spO}_{2}$ was significantly correlated with FEV1 ( $r=-546$, $P<0.001)$ and peak flow $(r=0.44, P<0.001)$ and mean spO2 was correlated with FEV1 $(r=0.57, \mathrm{P}<0.001), \mathrm{FVC}(\mathrm{r}=-0.51, \mathrm{P}<0.001)$ and weakly with peak flow $(r=0.28, P=0.028)$. The three spirometry parameters (FEV1, FVC, and peak flow) were included in the multiple linear regressions with the 6MWD parameters. FVC was significant determinants of the HR $(P<0.001, \beta=-0.69)$. Peak flow determinants of the walking distance $(P<0.001, \beta=0.86)$. FEV1 
determinants of the $\min \mathrm{spO}_{2}(P=0.009, \beta=-0.1)$ and $\mathrm{FEV} 1$ weakly with mean $\mathrm{spO}_{2}(\mathrm{P}=0.016, \beta=0.06)$ (Table 4).

\section{Discussion}

In the present study, FVC and PEF mean differences were significant; however, as expected, the difference in the means was higher in group A compared to the group B, which is consistent with the results of a study by Tanriover [10]

Evaluation of respiratory performance based on 6MWT parameters showed a greater difference in the means of $\mathrm{spO}_{2}$ and distant walked in the group A compared to the second group, revealing a discrepancy between the results of this study and a study by Savci et al [11]. This discrepancy between the results of these two studies shows the need for further studies in this respect.

The HR showed inverse correlations with FVC in multiple linear regressions, which suggested patients with greater restrictive constraints more HR during the walking test. Also patients with less walking distance had greater restrictive constraints. Minimum and mean $\mathrm{spO}_{2}$ also showed correlations with FEV1, which suggested that patients with greater restrictive constraints had less min and mean spO2.these results, had consistency with Chen in Taiwan among patients with chronic obstructive pulmonary disease (COPD) [12].

\section{Conclusion}

The results of this study, based on the evaluation of parameters of respiratory performance, showed that preoperative respiratory physiotherapy can have a positive effect on the improvement and quality of respiratory performance of patients undergoing open cardiac surgery. Further evaluations are necessary in relation to the sensitivity and specificity of $6 \mathrm{mwt}$ parameters alone in the evaluation of respiratory performance.

\section{Ethics approval}

The local Ethics Committee of Tabriz University of Medical Sciences (with a code of 2272/4/5) approved this study. All participants gave written informed consent before data collection began.

\section{Conflict of interest: none declared.}

\section{References}

1. Tenling A, Hachenberg T, Tyden H, Wegenius G, Hedenstierna G. Atelectasis and gas exchange after cardiac surgery. Anesthesiology 1998; 89: 371-378. (doi: 10.1097/00000542-199808000-00013).

2. Wynne R, Botti M. Postoperative pulmonary dysfunction in adults after cardiac surgery with cardiopulmonary bypass: clinical significance and implications for practice. Am J Crit Care 2004; 13(5): 384-393. (PMID: 15470854)

3. Aida $\mathrm{N}$, Shibuya $\mathrm{M}$, Inoue $\mathrm{T}$. Respiratory muscle stretch gymnastics in patients with post coronary artery bypass grafting pain: impact on respiratory muscle function, activity, and mood and exercise capacity. J Med Dent Sci 2002; 49: 157-170. (PMID: 12641387)

4. Brasher PA, McClelland KH, Denehy L, Story I. Does removal of deep breathing exercises from a physiotherapy program including preoperative education and early mobilization after cardiac surgery alter patient outcomes? Aust J Physiother 2003; 49: 165-173. (PMID: 12952516)

5. Rajaei S, Dabbagh A. Risk Factors for postoperative respiratory mortality and morbidity in patients undergoing coronary artery bypass grafting. Anesthesiology and Pain Medicine 2012; 2(2): 60-65. (doi: 10.5812/aapm.5228)

6. Castillo R, Haas A. Chest physical therapy: comparative efficacy of preoperative and postoperative in the elderly. Arch Phys Med Rehabil 1985; 66: 376-379. (PMID: 4004535)

7. Herdy $A H$, Vila A, Ribeirio JP. Pre and postoperative cardiopulmonary rehabilitation in hospitalized patients undergoing coronary artery bypass surgery. Phys Ned Rehabil 2008; 87: 714-719. (PMID: 18716482)

8. Miller MR, Hankinson J, Brusasco V, Burgos F, Casaburi R, Coates A, et al. Standardisation of spirometry. Eur Respir J 2005; 26: 319-338. (doi: 10.1136/thx.2006.061648)

9. Takigawa N, Tada A, Soda R, Date H, Yamashita M, Endo S, et al. Distance and oxygen desaturation in 6-min walk test predict prognosis in COPD patients. Respire Med 2007; 101: 561-567. (PMID: 16899358) (doi: 10.1016/j.rmed.2006.06.017)

10. Tanriover B, Carlton D, Saddekni S. Effects of deep breathing exercises after coronary artery bypass surgery. Rehabil and Phys 2000; 57: 2151 2155.

11. Savcı S, Sakınç S, İnal İnce D, Arikan H, Can Z, Buran Y, Kuralay E. Active cycle of breathing techniques and incentive spirometer in coronary artery bypass graft surgery. Fizyoter Rehabil 2006; 17: 61-69.

12. Chen SH, Wu YT, Lin JJ, Lee CN, Huanq CY, Chiang LL. The correlations of the six-minute walk test and respiratory functions in chronic obstructive pulmonary disease patients with chronic hypercapnia. J ExpClin Med 2012; 4(1): 47-51. (doi: 10.1016/j.jecm.2011.11.008)

\section{Authors:}

Seyed Kazem Shakuri - PhD, Professor, Physical Medicine and Rehabilitation Research Center, Tabriz University of Medical Science, Tabriz, Iran;

Yaghoub Salekzamani - PhD, Associate Professor, Physical Medicine and Rehabilitation Research Center, Tabriz University of Medical Science, Tabriz, Iran;

Ali Taghizadieh - Postdoc, Assistant Professor, Tuberculosis and Lung Research Center, Tabriz University of Medical Science, Tabriz, Iran;

Hamed Sabbagh-Jadid - MD, Physical Medicine and Rehabilitation Research Center, Tabriz University of Medical Science, Tabriz, Iran;

Jamal Soleymani - PhD, Physical Medicine and Rehabilitation Research Center, Tabriz University of Medical Science, Tabriz, Iran;

Leyla Sahebi - PhD student, Tuberculosis and Lung Disease Research Center, Tabriz University of Medical Science, Tabriz, Iran. 